\title{
I.d) Selected videos
}

V1

\section{APPROACH TO THE PORTA HEPATICUS DURING CYTROREDUCTIVE SURGERY - TECHNICAL CONSIDERATIONS}

\begin{abstract}
A.Sardi (USA)
Peritoneal carcinomatosis requires extensive cytoreductive surgery with emphasis on complete cytoreduction in order to extend patient survival. Frequent areas of tumor involvement include the lesser sac and the porta hepaticus. This video discusses the technical approach to the porta hepaticus to ensure complete cytoreduction.
\end{abstract}

V2

I. URINARY RECONSTRUCTIONS AFTER CRS+HIPEC. URETER RECONSTRUCTIONS

\section{P. Barrios, I. Ramos, O. Crusellas, D. Sabia (Spain).}

Tumor involvement of the urinary tract occurs with some frequency in $\mathrm{PC}$ and has represented a limitation for CRS+HIPEC. In 1,000 CRS+HIPEC procedures performed by the peritoneal carcinomatosis program of Catalonia over a period of 11 years, we performed 64 resections of some portion of the urinary tract. In 9 cases the renal pelvis or the upper half of the ureter was affected and in 55 cases the distal ureter土the urinary bladder was affected. This first video shows the indications and techniques used to reconstruct the proximal, middle and distal ureter with bladder preservation: direct ureteral t-t reconstruction, ureteral crosslinking, tubulized small bowel segment interposition, appendix interposition and direct ureterovesical anastomosis.

\section{V3}

TECHNIC TO ACHIEVE OPTIMAL CYTROREDUCTIVE SURGERY IN PERITONEAL CARCINOMATOSIS TECHNIQUES OF SYSTEMATIC TOTAL PERITONECTOMY

\section{Kr Ashwin (India).}

At laparotomy the extent of peritoneal carcinomatosis was recorded according to the peritoneal cancer index $(\mathrm{PCl})$. Aggressive surgical cytoreduction was then undertaken to leave the patient with no visible disease. The main characteristic of our institution's approach consists of performing systematic total peritonectomy i.e (both macroscopically involved and normal surfaces), by means seven of parietal peritonectomy procedures (right diaphragmatic peritonectomy, left diaphragmatic peritonectomy, pelvic peritonectomy, glisson capsulectomy, parietal anterior peritonectomy, greater omentectomy, and lesser omentectomy.) Small and scattered localizations on the visceral surface were resected by local excision/electrocoagulation. In case of massive and/or deeply infiltrating disease, visceral resections were performed, including cholecystectomy, splenectomy, sigmoid, right or total colectomy, and hysterectomy with salpingo-oophorectomy in women. 
V4

\section{CIRCONFERENTIEL PARIETAL AND MESENTERIC COMPLETE PERITONECTOMY WITH CO2}

\section{PNEUMATIZATION}

\section{P. Barrios, I. Ramos, O. Crusellas, D. Sabia (Spain).}

Malignant neoplasms of the peritoneal surface such as peritoneal mesothelioma and primary peritoneal carcinoma may affect the mesenteric leaves of the small intestine in a diffuse manner without compromising mesenteric vascularization. It is a laborious surgical procedure that can be performed safely and more quickly by injecting $\mathrm{CO}_{2}$ into the subperitoneal space of both mesenteric sheets through the insufflation devices used in laparoscopy. Once the mesenteric peritoneum of the vascular network is detached, it is completely eliminated with digitoclasis or the use of the electrocautery. In this video we present the indications and surgical technique used in 7 cases of the 1,000 CRS+HIPEC procedures performed by our peritoneal carcinomatosis program of Catalonia over a period of 